\title{
Meta-analysis of rosuvastatin efficacy in prevention of contrast-induced acute kidney injury
}

This article was published in the following Dove Press journal:

Drug Design, Development and Therapy

\author{
Jing Zhang' \\ Ying Guo' \\ Qi Jin' \\ Li Bian² \\ Ping Lin ${ }^{3}$
}

'Department of Nephrology, China-Japan Union Hospital of Jilin University, Changchun, People's Republic of China; ${ }^{2}$ Department of Radiotherapy, The Second Hospital of Jilin University, Changchun, People's Republic of China; ${ }^{3}$ Department of Nephrology, The First Hospital of Jilin University, Changchun, People's Republic of China
Correspondence: Li Bian

Department of Radiotherapy,

The Second Hospital of Jilin University,

No 218 Ziqiang Street, Changchun,

I3004I, People's Republic of China

Tel +86 I55 26852 I 58

Email 1005573353@qq.com

Ping Lin

Department of Nephrology,

The First Hospital of Jilin University,

No 7 I Xinmin Street, Changchun,

I3002I, People's Republic of China

Tel +86 I594306 443 I

Email linping1027@I63.com
Background: Contrast-induced nephropathy $(\mathrm{CIN})$ is a complication after the intravascular administration of a contrast medium injection. Previous studies have investigated statins as therapy for $\mathrm{CIN}$ due to its positive results in the prevention of contrast-induced acute kidney injury (CI-AKI). Nevertheless, the beneficial effects of rosuvastatin pretreatment in preventing CIN in patients with acute coronary syndromes still remain controversial. In this study, we performed a meta-analysis of randomized controlled trials (RCTs) to evaluate the beneficial impact of rosuvastatin in the prevention of CI-AKI in acute coronary syndrome patients.

Methods: PubMed, Embase, and Cochrane library were searched, for RCTs, updated on January 2018. The method was to evaluate rosuvastatin prior to angiography for the prevention of CI-AKI in patients undergoing coronary angiography, of which the main outcome was the incidence of CIN.

Results: A total of five RCTs were included in this analysis. Patients treated with rosuvastatin prior to invasive angiography had a significantly lower incidence of CI-AKI than controls (odds ratio [OR]: $0.53,95 \% \mathrm{CI}: 0.40-0.71, P<0.0001$ ). Moreover, the subgroup analysis also showed that the benefit of rosuvastatin for patients with chronic kidney disease (OR: 0.49 , 95\% CI: $0.26-0.92, P=0.03$ ) and diabetes mellitus (OR: $0.56,95 \% \mathrm{CI}: 0.38-0.83, P=0.004$ ) which was consistent in compared with the respective control groups.

Conclusion: The findings of this meta-analysis suggest that the preoperative rosuvastatin treatment significantly reduces the risk of renal insufficiency of CIN in at-risk patients with chronic kidney disease or diabetes mellitus. Additional studies are needed to identify at-risk patients, provide optimum dose peri-procedural treatment, and reduce the incidence of CIN.

Keywords: contrast-induced nephropathy, coronary angiography, rosuvastatin, meta-analysis

\section{Introduction}

Contrast-induced nephropathy (CIN) is characterized by acute impairment of renal function after contrast exposure. ${ }^{1}$ Patients with acute coronary syndrome (ACS) undergoing primary percutaneous intervention (p-PCI) are at a higher risk of developing contrast-induced acute kidney injury (CI-AKI). ${ }^{2}$ Moreover, the incidence of contrast nephropathy was associated with patient characteristics that are risk factors for this disease. ${ }^{3,4}$ As CI-AKI is associated with increased short- and long-term morbidity and mortality, nonfatal cardiovascular events, and a longer hospital stay, ${ }^{5}$ the adoption of optimal therapeutic strategies is needed to prevent CIN, offering an opportunity to reduce patient morbidity and mortality.

In clinical practice, a number of studies have shown that the pleiotropic properties of statins exert a beneficial impact in the prevention of contrast nephropathy. ${ }^{6}$ Apart from their cholesterol-lowering effects, statins have anti-inflammatory, antithrombotic, and antioxidative properties and may exercise nephron-protective action, thereby improving 
the endothelial function and reducing vascular inflammation and oxidative stress. ${ }^{5}$ These favorable activities have a direct preventive effect against the development of CI-AKI. ${ }^{7,8}$ However, the results concerning the efficacy of statin therapy in the prevention of CI-AKI are inconsistent. ${ }^{6,9,10}$

The hydrophilic form of the statin rosuvastatin may have better potential for prevention of CIN than other statin forms, probably owing to its longer plasma half-life and stronger anti-inflammatory effect. Previous randomized controlled trials (RCTs) focused mainly on the application of the lipophilic form of rosuvastatin for the prevention of CIN, but conflicting results have been reported.

Therefore, in this study, we performed a meta-analysis of RCTs to evaluate the efficacy of rosuvastatin pretreatment for the prevention of CIN as compared with placebo treatment.

\section{Methods}

\section{Search strategy}

To find all related articles, two investigators independently searched electronic databases (PubMed, Embase, and Cochrane Library) up to January 2018. The keywords "rosuvastatin" and "contrast-induced acute kidney injury," as well as relevant Medical Subject Heading terms were used in the search. We also checked the reference lists of all articles for additional eligible studies.

\section{Eligibility criteria}

To be included in the meta-analysis, the studies had to meet the following criteria: 1) RCTs comparing rosuvastatin pretreatment with placebo treatment in patients undergoing PCI; 2) they provided information about CI-AKI; 3) the outcome of interest was the incidence of CIN; 4) each paper had to be written in English.

\section{Quality assessment}

Two investigators independently rated the quality of the eligible RCTs. We choose the risk of bias items for RCTs recommended by The Cochrane Handbook for Systematic Reviews of Interventions. ${ }^{32}$

\section{Data extraction}

Two authors independently extracted the relevant data from each article. Any disagreement was resolved by consensus. The data extracted from the eligible studies included the following information: name of the first author, year of publication, characteristics of the trials, number of study patients, mean age, mean baseline SCr, and definition of $\mathrm{CIN}$ in each trial.

\section{Statistical analysis}

All outcome comparisons were conducted and estimates calculated using the Review Manager version 5.3 software (Revman; The Cochrane Collaboration, Oxford, UK). Odds ratios (ORs) and their 95\% CIs were calculated to assess the CIN events and make comparisons between the rosuvastatin and the control groups. The ORs and their 95\% CIs were also mentioned in the summary statistics in the pooled subgroup analysis for patients with chronic kidney disease (CKD) or diabetes mellitus (DM).

A sensitivity analysis was also performed to examine the overall impact of heterogeneity on meta-analysis results. The $I^{2}$ test was employed to evaluate the heterogeneity of the results. ${ }^{11}$ An $I^{2}$-value $\geq 50 \%$ was considered to indicate moderate and high heterogeneity, whereas an $I^{2}$-value $<50 \%$ showed low heterogeneity. ${ }^{12}$ A $P$-value $<0.05$ was considered statistically significant.

\section{Results \\ Overview of literature search and study characteristics}

A total of 213 studies were identified and reviewed according to the criteria described in the methods, of which 10 publications were evaluated in more detail, but some did not provide information on the endpoint outcomes.

Thus, a total of five RCTs were finally included in the present analysis. ${ }^{13-17}$ The search process is illustrated in Figure 1. All included studies in this meta-analysis were considered to be at least of moderate quality. Detailed information on the medication protocols of the eligible studies is presented in Table 1.

\section{Effect of rosuvastatin on the incidence of $\mathrm{Cl}-\mathrm{AKI}$}

The data pooled from all five articles included in our research showed that the risk of CI-AKI (OR: $0.53,95 \% \mathrm{CI}$ : $0.40-0.71, P<0.0001)$ in the atorvastatin treatment group was lower than that in the control group (Figure 2).

\section{Subgroup analysis of the effect of rosuvastatin on the incidence of $\mathrm{Cl}-\mathrm{AKI}$}

Rosuvastatin reduced also the risk of CI-AKI in patients with CKD (OR: 0.45, 95\% CI: 0.21-0.95, $P=0.04$ ) (Figure 3 ) and DM (OR: $0.56,95 \%$ CI: 0.38-0.83, $P=0.004$ ) (Figure 4), as compared with that in the placebo group. 


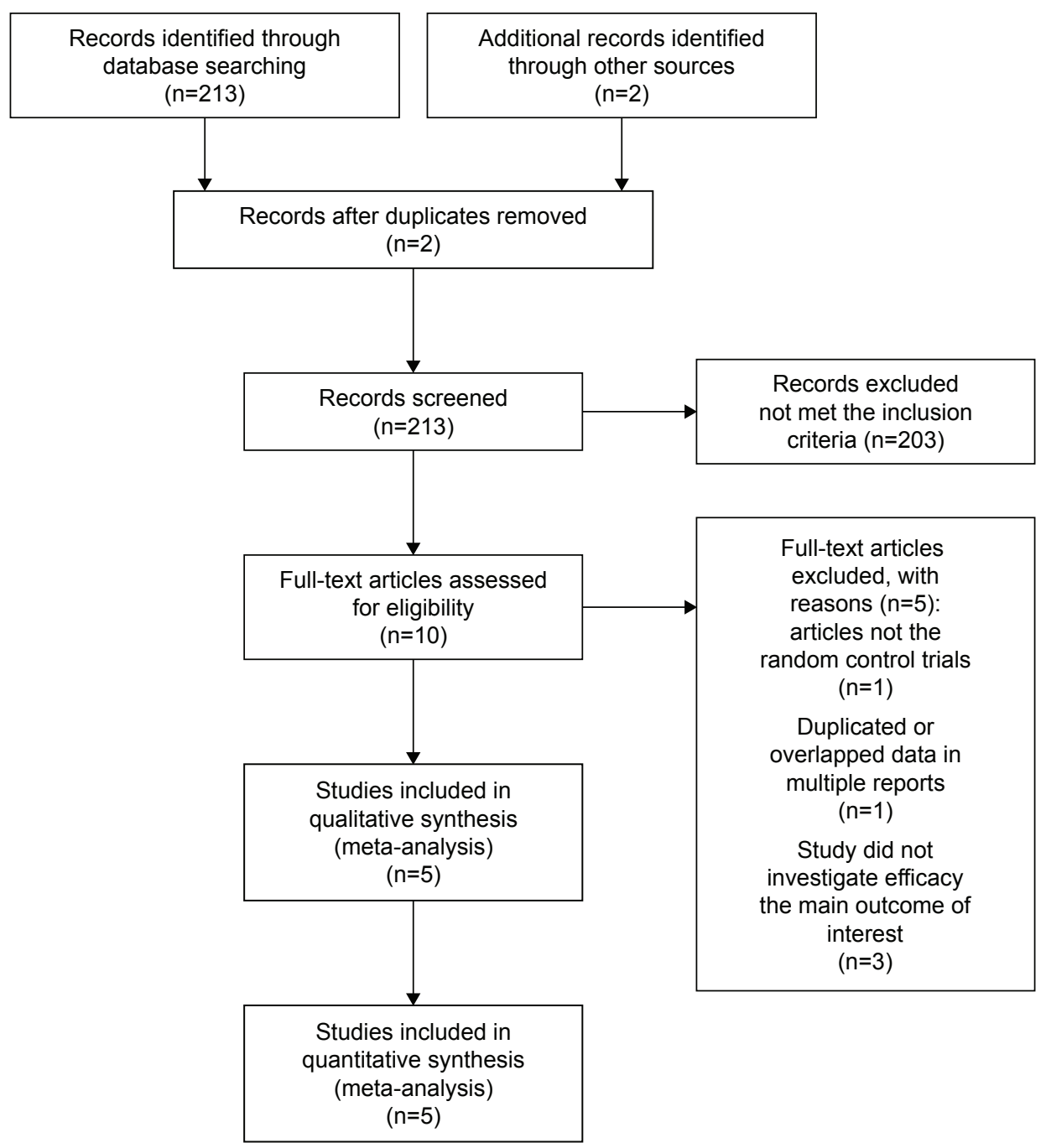

Figure I PRISMA flow chart of the selection process used to identify studies eligible for pooling.

Abbreviation: RCT, randomized controlled trial.

\section{Discussion}

The pathophysiological mechanism of CIN is still not entirely clear. Multiple mechanisms may be involved; in particular, adenosine, angiotensin, vasopressin, and endothelin secretion after contrast exposure is a logical cause for the reduction in the synthesis of nitric oxide and the induction of oxidative stress, causing hypoxia in the renal medulla. More recently, other renal damage mechanisms have been associated with the development of cellular lesions, necrosis, interstitial inflammation, and tubular injury. ${ }^{5,7}$ It is noteworthy that the elevated preprocedural concentration of the high-sensitivity CRP was found to increase the risk of CIN in patients who had underwent PCI. ${ }^{18}$

In addition to their lipid-lowering activities, statins are also known to downregulate the angiotensin receptor, limit the production of endothelial nitric oxide synthase, and have antithrombotic and anti-inflammatory properties, which altogether constitutes their pleiotropic effects. ${ }^{9}$ Due to these multidirectional activities, statins are considered to exert also a renoprotective effect and that the pretreatment with statin can affect the progression of the CI-AKI pathogenesis.

Basing on the beneficial clinical results observed in various subgroups of patients, we analyzed the additional functions of rosuvastatin which showed acute pleiotropic effects. ${ }^{19}$ In one of the studies included in our research, healthy patients without hyperlipidemia (low-density lipoprotein cholesterol levels: $<130 \mathrm{mg} / \mathrm{dL}$ ) but with elevated high-sensitivity CRP levels $(>2.0 \mathrm{mg} / \mathrm{L})^{20}$ and patients with estimated eGFR $<60 \mathrm{~mL} / \mathrm{min} / 1.73 \mathrm{~m}^{2}$ were found to be at the highest risk of CI-AKI. ${ }^{21}$ Furthermore, the short-term treatment with rosuvastatin was indicated to play a protective role by improving eGFR independently of the lipid fraction changes, 


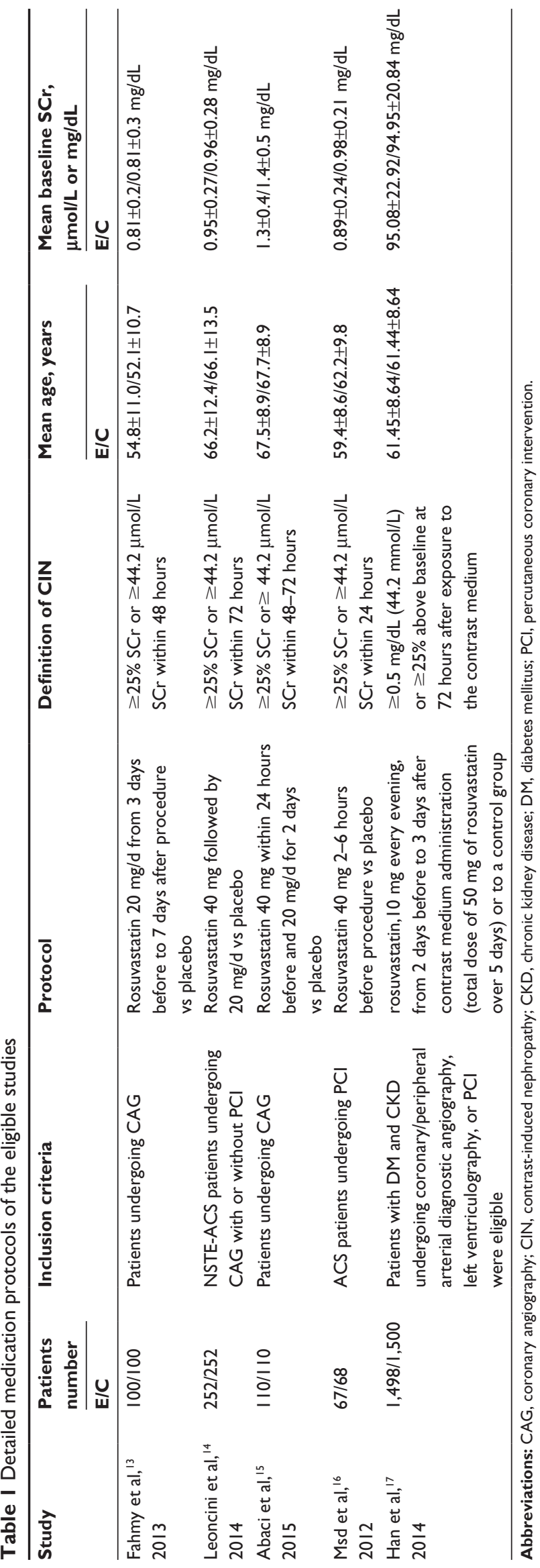

suggesting that the pleiotropic effect of rosuvastatin included also renoprotective functions..$^{22}$ The preventive impact of rosuvastatin on the magnitude of $\mathrm{CIN}$ reduction could be due to of its inhibitory influence on the synthesis of inflammatory mediators, a phenomenon observed in the JUPITER trial. ${ }^{20}$

Previous studies have shown the beneficial, preventive effects of statins against CI-AKI. For example, Leoncini et $\mathrm{al}^{14}$ established that rosuvastatin significantly reduced CI-AKI. However, Abaci et $\mathrm{al}^{15}$ found that the high-dose rosuvastatin treatment had no beneficial effects in decreasing the occurrence of CI-AKI in patients with suspected coronary artery disease.

Preexisting kidney disease is known to be the greatest risk for CI-AKI development, especially in patients with acute coronary syndrome because of their diminished renal reserve. ${ }^{23}$ Nevertheless, whether the short-term high-dose statin pretreatment in patients with stable coronary artery disease lowers the risk of CI-AKI and reduces the renal function still remains debatable, and thus additional research is needed to further elucidate this issue. ${ }^{24-26}$ Moreover, patients with DM are at the highest risk of CI-AKI due to the pathophysiologic changes caused by contrast materials, including increased production of reactive oxygen species, vascular endothelial injury, and dysregulated microcirculation. Thus, patients with CKD and DM are at increased risk of CI-AKI. ${ }^{27}$

In the current study, we found that the beneficial effects of rosuvastatin were consistent in patients with renal dysfunction and diabetes with a significant reduction of CI-AKI rate. The pathophysiology contributing to kidney injury in CIN is not completely understood. Given their multiple pleiotropic effects, statins are likely to exert a renoprotective effect and limit the injury of renal function over time. ${ }^{5}$ Preclinical studies have demonstrated that statins inhibit receptor-mediated endocytosis, which is responsible for the protein uptake in proximal tubular cells, in a dose-dependent manner. ${ }^{28,29}$

The latest meta-analysis published suggested that the moderate- or high-dose rosuvastatin treatment may have preventive effects against CIN in patients undergoing coronary angiography or PCI. As the data available on this dose-dependent influence are limited, in this meta-analysis, we did not assess the impact of the potentially hazardous high-dose rosuvastatin pretreatment. ${ }^{30}$

The main relative strength of our analysis as compared with previous meta-analyses on the same topic is the inclusion and analysis of the results of randomized controlled trials published in English. Nonetheless, several inherent flaws of the study should not be ignored as that might have led to potential bias. First, data were pooled from a number 


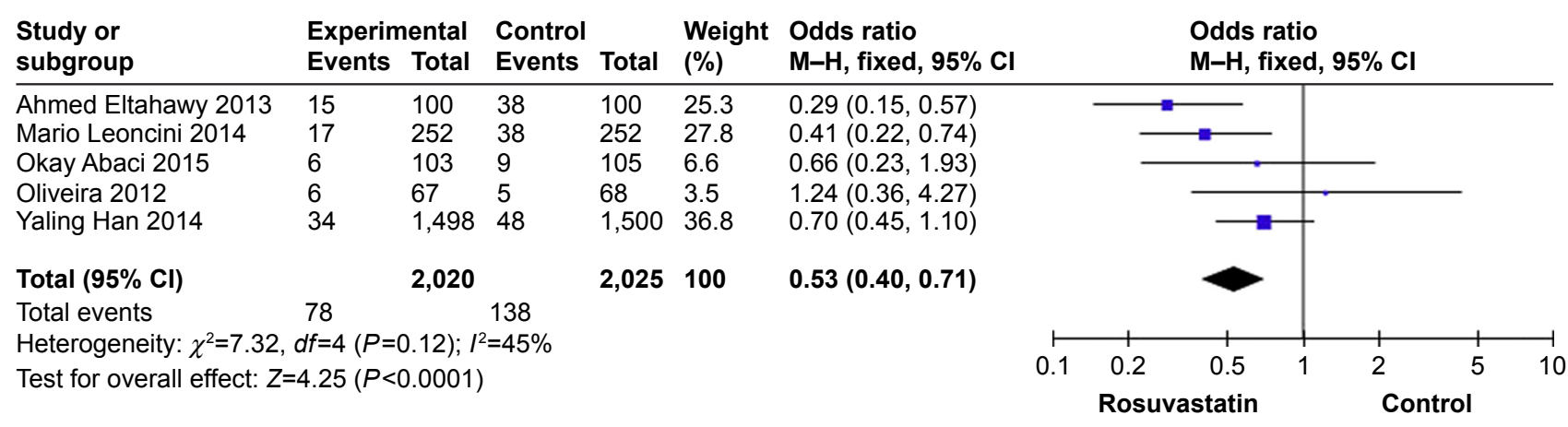

Figure 2 Effect of rosuvastatin on the incidence of $\mathrm{Cl}-\mathrm{AKI}$.

Abbreviations: $\mathrm{Cl}-\mathrm{AKI}$, contrast-induced acute kidney injury; OR, odds ratio.

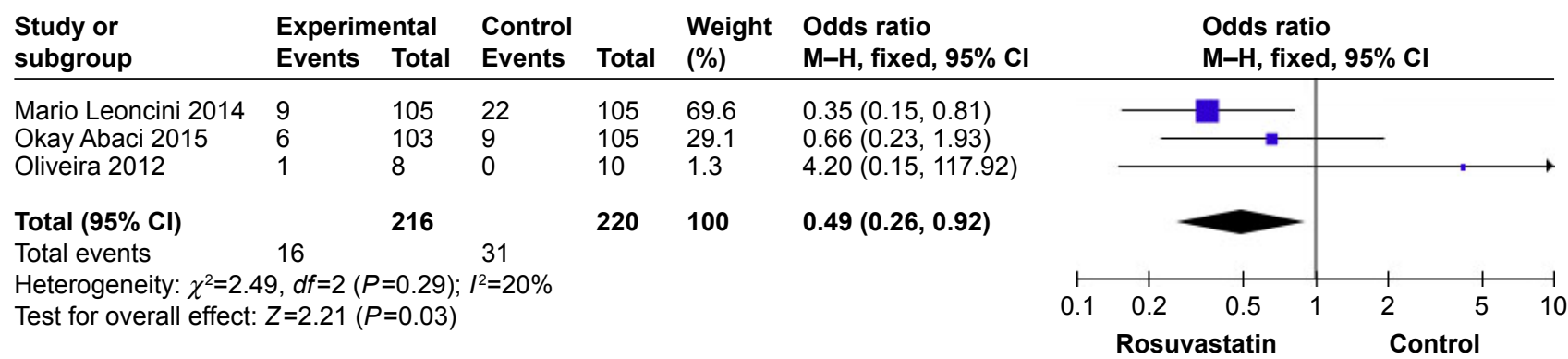

Figure 3 Influence of rosuvastatin treatment on the incidence of $\mathrm{Cl}-\mathrm{AKI}$ in patients with CKD.

Abbreviations: $\mathrm{Cl}-\mathrm{AKI}$, contrast-induced acute kidney injury; CKD, chronic kidney disease; OR, odds ratio.

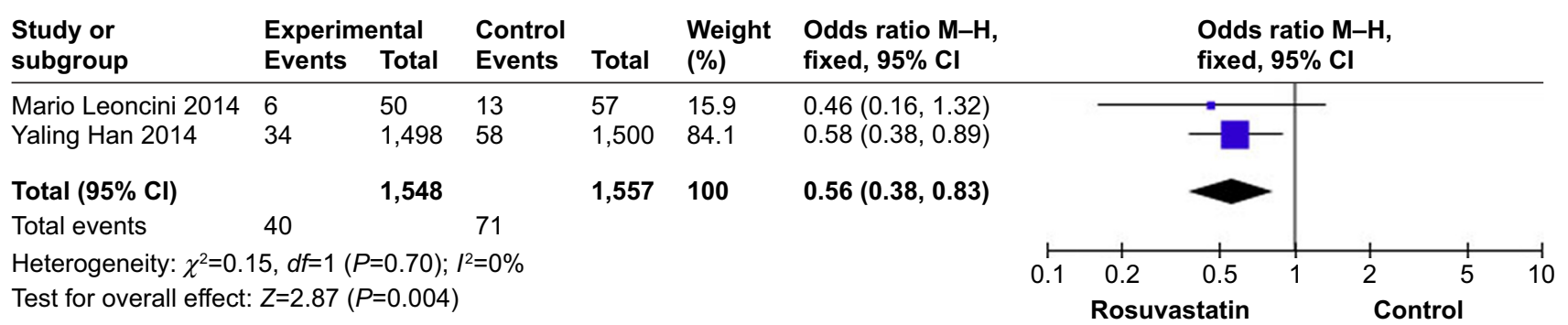

Figure 4 Impact of rosuvastatin administration on the incidence of $\mathrm{Cl}-\mathrm{AKI}$ in patients with DM.

Abbreviations: $\mathrm{Cl}-\mathrm{AKI}$, contrast-induced acute kidney injury; DM, diabetes mellitus; OR, odds ratio.

of RCTs with different inclusion criteria with insignificant heterogeneity among the included studies. Second, patients might have had differential responses to treatment with different doses of rosuvastatin. Third, the entire meta-analysis is based upon 5 RCTs, of which only three related to CKD and two related to DM, which limits the ability to statistically analyze the effect of rosuvastatin on the incidence of interested outcomes, so future research should be conducted to identify these subgroups.

Contrast administration is well-established in the literature as a risk factor for CI-AKI. The incidence of CI-AKI varies widely depending on the patient cohorts evaluated, definition criteria used, and preventive strategies adopted. ${ }^{31}$
In conclusion, the current evidence indicates that rosuvastatin prevents the occurrence of CIN in patients after an episode of CI-AKI, especially in high-risk patients with DM or CKD.

The best approaches established so far to prevent CIN are to identify at-risk patients, provide adequate therapeutic doses, and minimize the amount of contrast administered. However, the actual mechanism by which statins may lower the risk of contrast nephropathy remains uncertain. Therefore, further research is needed to better understand their underlying mechanisms of action.

\section{Disclosure}

The authors report no conflicts of interest in this work. 


\section{References}

1. Solomon R, Dauerman HL. Contrast-induced acute kidney injury. Circulation. 2010;122(23):2451-2455.

2. Senoo T, Motohiro M, Kamihata H, et al. Contrast-induced nephropathy in patients undergoing emergency percutaneous coronary intervention for acute coronary syndrome. Am J Cardiol. 2010;105(5):624-628.

3. Rihal CS, Textor SC, Grill DE, et al. Incidence and prognostic importance of acute renal failure after percutaneous coronary intervention. Circulation. 2002;105(19):2259-2264.

4. Sudarsky D, Nikolsky E. Contrast-induced nephropathy in interventional cardiology. Int J Nephrol Renovasc Dis. 2011;4:85-99.

5. Mccullough PA. Radiocontrast-induced acute kidney injury. Nephron Physiol. 2008;109(4):p61-p72.

6. Pappy R, Stavrakis S, Hennebry TA, Abu-Fadel MS. Effect of statin therapy on contrast-induced nephropathy after coronary angiography: a meta-analysis. Int J Cardiol. 2011;151(3):348-353.

7. Tumlin J, Stacul F, Adam A, et al. Pathophysiology of Contrast-Induced Nephropathy. Am J Cardiol. 2006;98(6):14-20.

8. Giusti-Paiva A, Martinez MR, Felix JV, et al. Simvastatin decreases nitric oxide overproduction and reverts the impaired vascular responsiveness induced by endotoxic shock in rats. Shock. 2004;21(3):271-275.

9. Patti G, Ricottini E, Nusca A, et al. Short-term, high-dose Atorvastatin pretreatment to prevent contrast-induced nephropathy in patients with acute coronary syndromes undergoing percutaneous coronary intervention (from the ARMYDA-CIN [atorvastatin for reduction of myocardial damage during angioplasty - contrast-induced nephropathy]) trial. Am J Cardiol. 2011;108(1):1-7.

10. Zhang L, Zhang L, Lu Y, et al. Efficacy of statin pretreatment for the prevention of contrast-induced nephropathy: a meta-analysis of randomised controlled trials. Int J Clin Pract. 2011;65(5):624-630.

11. Higgins JP, Thompson SG. Quantifying heterogeneity in a metaanalysis. Stat Med. 2002;21(11):1539-1558.

12. Higgins JP, Thompson SG, Deeks JJ, Altman DG. Measuring inconsistency in meta-analyses. BMJ. 2003;327(7414):557-560.

13. Fahmy M, Eltahawy A, Enany BE. TCTAP A-037 Role of Rosuvastatin Pretreatment in Prevention of Contrast Induced Nephropathy in Patients Undergoing Coronary Angiography. J Am Coll Cardiol. 2014; 63(12):S11.

14. Leoncini M, Toso A, Maioli M, et al. Early high-dose rosuvastatin and cardioprotection in the protective effect of rosuvastatin and antiplatelet therapy on contrast-induced acute kidney injury and myocardial damage in patients with acute coronary syndrome (PRATO-ACS) study. Am Heart J. 2014;168(5):792-797.

15. Abaci O, Arat Ozkan A, Kocas C, et al. Impact of Rosuvastatin on contrast-induced acute kidney injury in patients at high risk for nephropathy undergoing elective angiography. Am J Cardiol. 2015;115(7): 867-871.

16. Msd O, Bomfim Araujo Martins K, Ribamar Costa J, et al. Impact on Renal Function of Rosuvastatin Preload Prior to Elective Percutaneous Coronary Intervention in Chronic Statin Users. Revista Brasileira de Cardiologia Invasiva. 2012;20(3):303-308.

17. Han Y, Zhu G, Han L, et al. Short-term rosuvastatin therapy for prevention of contrast-induced acute kidney injury in patients with diabetes and chronic kidney disease. J Am Coll Cardiol. 2014;63(1): $62-70$.

Drug Design, Development and Therapy

\section{Publish your work in this journal}

Drug Design, Development and Therapy is an international, peerreviewed open-access journal that spans the spectrum of drug design and development through to clinical applications. Clinical outcomes, patient safety, and programs for the development and effective, safe, and sustained use of medicines are the features of the journal, which
18. Briasoulis A, Pala M, Telila T, et al. Statins and contrast-induced nephropathy: a systematic review and meta-analysis. Curr Pharm Des. 2017.

19. Liuni A, Luca MC, Gori T, Parker JD. Rosuvastatin prevents conduit artery endothelial dysfunction induced by ischemia and reperfusion by a cyclooxygenase-2-dependent mechanism. J Am Coll Cardiol. 2010;55(10):1002-1006.

20. Ridker PM, Danielson E, Fonseca FA, et al. Rosuvastatin to prevent vascular events in men and women with elevated C-reactive protein. The New England Journal of Medicine. 2008;359(21):2195-2207.

21. Ridker PM, Macfadyen J, Cressman M, Glynn RJ. Efficacy of rosuvastatin among men and women with moderate chronic kidney disease and elevated high-sensitivity C-reactive protein: a secondary analysis from the JUPITER (Justification for the Use of Statins in Prevention-an Intervention Trial Evaluating Rosuvastatin) trial. J Am Coll Cardiol. 2010;55(12):1266-1273.

22. Vidt DG, Harris S, Mctaggart F, Ditmarsch M, Sager PT, Sorof JM. Effect of short-term rosuvastatin treatment on estimated glomerular filtration rate. Am J Cardiol. 2006;97(11):1602-1606.

23. Marenzi G, Lauri G, Assanelli E, et al. Contrast-induced nephropathy in patients undergoing primary angioplasty for acute myocardial infarction. J Am Coll Cardiol. 2004;44(9):1780-1785.

24. Jo SH, Koo BK, Park JS, et al. Prevention of radiocontrast mediuminduced nephropathy using short-term high-dose simvastatin in patients with renal insufficiency undergoing coronary angiography (PROMISS) trial - a randomized controlled study. Am Heart J. 2008;155499(3): e491-e498.

25. Toso A, Maioli M, Leoncini M, et al. Usefulness of atorvastatin ( $80 \mathrm{mg}$ ) in prevention of contrast-induced nephropathy in patients with chronic renal disease. Am J Cardiol. 2010;105(3):288-292.

26. Quintavalle C, Fiore D, de Micco F, et al. Impact of a high loading dose of atorvastatin on contrast-induced acute kidney injury. Circulation. 2012;126(25):3008-3016.

27. Mccullough PA, Wolyn R, Rocher LL, Levin RN, O’Neill WW. Acute renal failure after coronary intervention: incidence, risk factors, and relationship to mortality. Am J Med. 1997;103(5):368-375.

28. Agarwal R. Statin induced proteinuria: renal injury or renoprotection? J Am Soc Nephrol. 2004;15(9):2502-2503.

29. Harper CR, Jacobson TA. Managing dyslipidemia in chronic kidney disease. J Am Coll Cardiol. 2008;51(25):2375-2384.

30. Liang M, Yang S, Fu N. Efficacy of short-term moderate or high-dose rosuvastatin in preventing contrast-induced nephropathy: A metaanalysis of 15 randomized controlled trials. Medicine. 2017;96(27): e7384.

31. Mehran R, Aymong ED, Nikolsky E, et al. A simple risk score for prediction of contrast-induced nephropathy after percutaneous coronary intervention: development and initial validation. J Am Coll Cardiol. 2004;44(7):1393-1399.

32. Higgins JPT, Green S (editors) [wepage on Internet]. Cochrane Handbook for Systematic Reviews of Interventions Version 5.1.0 [updated March, 2011]. Available from: www.handbook.cochrane.org. Accessed October 19, 2018.

\footnotetext{
Submit your manuscript here: http://www.dovepress.com/drug-design-development-and-therapy-journal
}

has also been accepted for indexing on PubMed Central. The manuscript management system is completely online and includes a very quick and fair peer-review system, which is all easy to use. Visit http://www.dovepress.com/testimonials.php to read real quotes from published authors. 